Journal of Applied Pharmaceutical Science Vol. 5 (11), pp. 010-014, November, 2015

Available online at http://www.japsonline.com

DOI: $10.7324 / \mathrm{JAPS} .2015 .501102$

ISSN 2231-3354 (cc) BY-NC-SA

\title{
Acrylic hydrogels modified with bee pollen for biomedical applications
}

\author{
Bożena Tyliszczak ${ }^{1}$, Dorota Walczyk ${ }^{1 *}$, Sławomir Wilczyński \\ ${ }^{1}$ Cracow University of Technology, Department of Chemistry and Technology of Polymers, 24 Warszawska St., 31 -155 Krakow, Poland. \\ ${ }^{2}$ School of Pharmacy with the Division of Laboratory Medicine in Sosnowiec, Medical University of Silesia in Katowice, Department of Basic Biomedical \\ Science, Kasztanowa St. 3, Sosnowiec, Poland.
}

\begin{tabular}{l} 
ARTICLE INFO \\
\hline Article history: \\
Received on: 02/09/2015 \\
Revised on: 26/09/2015 \\
Accepted on: 20/10/2015 \\
Available online: $27 / 11 / 2015$ \\
\hline Key words: \\
acrylic hydrogels, bee pollen, \\
swelling ability, simulated \\
body fluids
\end{tabular}

\begin{abstract}
The focus of this paper is to present the effect of bee pollen on the structure and properties of acrylic hydrogels, often used as materials for wound dressings. Honey, propolis and bee pollen have been very well known for their extraordinary healing and medical properties for thousands of years. Ancient Egyptians, Greeks and Romans have reportedly used bee pollen as a drug, especially for a number of skin diseases and lesions. Acrylic hydrogels are, on the other hand, rather recently discovered materials used for novel wound dressings. The idea of our research was to combine a synthetic polymer matrix with bee pollen, in order to obtain innovative materials which could be used as wound dressings exhibiting new properties. The obtained acrylic hydrogels containing bee pollen were investigated towards swelling ability in distilled water and different fluids, including pseudoextracellular fluid, simulated body fluid and Ringer's solution. Moreover, incubation in water and simulated body fluids (SBF) and Ringer's solution was carried out. The morphologies of bee pollen and obtained acrylic hydrogels modified with bee pollen were characterized by means of scanning electron microscopy (SEM). The chemical structure of the polymer matrix was confirmed by ATR-FT-IR spectra.
\end{abstract}

\section{INTRODUCTION}

Acrylic hydrogels are polymeric matrices with a threedimensional structure, which is characterized by an extraordinarily high swelling degree in water and other fluids. Moreover, appropriate synthesis allows them to be biocompatible, biodegradable and bioactive - all are traits of good biomaterials (Peppas and Hilt 2006; Serra et al., 2009). Although these materials have a vast number of possible applications, we focus solely on their use as wound dressings, which are especially used in treatment of slowly healing and chronic wounds, such as decubitus ulcers, venous ulcers, diabetic foot ulcers, as well as second-degree burns and post-operative wounds (Queen et al., 2004). Introduction of an active component into the polymer matrix would allow the hydrogel to exhibit additional properties, rendering it an even more suitable material for biomedical applications. Natural products are a promising source of new discoveries in the field of pharmacy and medicine,

\footnotetext{
* Corresponding Author

Dorota Walczyk, Cracow University of Technology, Department of

Chemistry and Technology of Polymers, 24 Warszawska St., 31-155

Krakow, Poland.Email:dorotawalczyk29@gmail.com
}

though a majority of them has been known for centuries. An excellent example is provided by apiproducts, such as honey, propolis and bee pollen, which have been known since the ancient times because of their unique properties. Bee pollen and propolis both exhibit a vast spectrum of biological and pharmacological properties, such as immunomodulatory, anti-inflammatory and antioxidant (Burdock, 1998; Catchpole et al., 2004; Kashkooli et al., 2011; Leja et al., 2007; Sforcin 2007; Sforcin and Bankova 2011). Bee pollen contains a variety of nutrients, such as amino acids, minerals, including phosphorus, potassium, magnesium, calcium, copper, manganese, iron and zinc, some trace minerals, all $B$ vitamins, including $B_{12}$, folic acid, panthothenic acid, rutin and lecithin (Almeida-Muradian et al., 2005; Human and Nicolson 2006; Kashkooli et al., 2011; Kroyer and Hegedus, 2001; Sforcin, 2007). It appears highly beneficial for human organism to profit from these bee products, and one may see a great potential of their use especially in healing and wound dressing materials, as they can provide additional anti-inflammatory activity, thus leading to shorter healing time. We have followed this lead and we have focused on modifying hydrogels with bee pollen, hoping that these would constitute very interesting materials for biomedical applications. 


\section{MATERIALS AND METHODS}

Acrylic acid (abbrv. AA; 99.5\%wt.), potassium hydroxide $(\mathrm{KOH}$, aq. solution, volumetric standard $1 \mathrm{M})$ and ammonium persulfate $\left(\left(\mathrm{NH}_{4}\right)_{2} \mathrm{~S}_{2} \mathrm{O}_{8} ; \quad \geq 98.0 \%\right.$ wt. $)$ were all purchased from Avantor Performance Materials Poland. Poly(ethylene glycol) diacrylate (abbrv. PEGDA, $\mathrm{M}_{\mathrm{n}}=700$ ) was purchased from Sigma-Aldrich and bee pollen (BP) was obtained from Apipol-Farma.

\section{Preparation}

The acrylic hydrogels modified with bee pollen (BP) were prepared by free-radical polymerization in solution in presence of a small amount of initiator $\left(1 \%\right.$ wt. $\left(\mathrm{NH}_{4}\right)_{2} \mathrm{~S}_{2} \mathrm{O}_{8}$ solution). Firstly, an appropriate amount of acrylic acid $(20 \mathrm{ml})$ was mixed with $40 \%$ wt. solution of potassium hydroxide $(16.5 \mathrm{ml})$. After reaching the ambient temperature $\left(25^{\circ} \mathrm{C}\right)$, bee pollen ( $2.5 \%$ wt. or $5 \% \mathrm{wt}$ ), initiator $(0.075 \% \mathrm{wt}$.) and crosslinking agent - poly(ethylene glycol) diacrylate (PEGDA, Mn $=575,4.5 \%$ wt.), were added.

The reacting mixture was mixed intensively on a magnetic stirrer at $60^{\circ} \mathrm{C}$ for $30 \mathrm{~min}$. Afterwards the mixture was poured into Petri dishes, which were left at $37^{\circ} \mathrm{C}$ for $24 \mathrm{~h}$. The compositions of formed acrylic hydrogels modified by appropriate amounts of bee pollen $0,2.5$ and $5 \% \mathrm{wt}$. respectively are shown in Table 1 presented below.

Table 1: The compositions of acrylic hydrogels modified by bee pollen.

\begin{tabular}{ccccc}
\hline Samples & $\begin{array}{c}\text { Acrylic acid } \\
\text { (ml) }\end{array}$ & $\begin{array}{c}\text { Bee pollen } \\
\text { (wt \%) }\end{array}$ & $\begin{array}{c}\text { PEGDA } \\
\mathbf{5 7 5} \text { (wt \%) }\end{array}$ & $\begin{array}{c}\text { APS - initiator } \\
\text { (wt \%) }\end{array}$ \\
\hline PAA & 20 & 0 & 4.5 & 0.075 \\
PAA & 20 & 2,5 & 4.5 & 0.075 \\
PAA & 20 & 5 & 4.5 & 0.05 \\
\hline
\end{tabular}

\section{Swelling behaviour}

The dried samples of obtained acrylic hydrogels, each weighing $0.5 \mathrm{~g}$, were immersed in the following fluids: water, $0.9 \%$ wt. $\mathrm{NaCl}$ solution, $0.9 \%$ wt. $\mathrm{CaCl}_{2}$ solution, Ringer's solution $\left(\mathrm{pH}=5 \div 7.50 ; \quad \mathrm{Na}^{+}=147.2 \mathrm{mmol} \cdot \mathrm{dm}^{-3}, \quad \mathrm{Cl}^{-}=\right.$ $155.7 \mathrm{mmol} \cdot \mathrm{dm}^{-3}, \quad \mathrm{~K}^{+}=4.0 \mathrm{mmol} \cdot \mathrm{dm}^{-3}, \mathrm{Ca}^{2+}=2.2 \mathrm{mmol} \cdot \mathrm{dm}^{-3}$ ) and simulated body fluid $(\mathrm{SBF}, \mathrm{pH}=7.40)$ and in pseudoextracellular fluid (PECF) $(\mathrm{pH}=7.4 \pm 0,2)$, simulating the wound fluids. The comparison of concentrations of ions such as: $\mathrm{Na}^{+}, \mathrm{K}^{+}$, $\mathrm{Ca}^{2+}, \mathrm{Mg}^{2+}, \mathrm{HCO}_{3}^{-}, \mathrm{Cl}^{-}, \mathrm{HPO}_{4}^{-}$and $\mathrm{SO}_{4}^{2-}$ that are present in accordingly: human plasma, SBF and PECF are summarized below in Table 2 (Kokubo and Takadama 2006, Vallet-Regí and Arcos 2008).

The swelling capacity (Q) was calculated according to the following formula:

$$
Q=\frac{W_{1}-W_{0}}{W_{0}}
$$

where: $\mathrm{W}_{1}$ and $\mathrm{W}_{0}$ refer to the weights $[\mathrm{g}]$ of the hydrogel in swollen and dried states, respectively.
Table 2: Human plasma and ions concentration $[\mathrm{mM}]$ of SBF and PECF (Kokubo and Takadama, 2006; Vallet-Regí and Arcos, 2008).

\begin{tabular}{|c|c|c|c|c|c|c|c|c|}
\hline Ions $\rightarrow$ & $\mathrm{Na}^{+}$ & $\mathbf{K}^{+}$ & $\mathrm{Ca}^{2+}$ & $\mathrm{Mg}^{2+}$ & $\mathrm{HCO}_{3}^{-}$ & $\mathrm{Cl}^{-}$ & $\mathrm{HPO}_{4}$ & $\mathrm{SO}_{4}{ }^{2}$ \\
\hline Medium & \multicolumn{8}{|c|}{$\left[\mathrm{mmol} \cdot \mathrm{dm}^{-3}\right]$} \\
\hline $\begin{array}{c}\text { Human } \\
\text { plasma } \\
\text { (total) }\end{array}$ & 142 & 5 & 2.5 & 1.5 & 27 & 103 & 1 & 0.5 \\
\hline SBF & 142 & 5 & 2.5 & 1.5 & 4.2 & 148 & 1 & 0,5 \\
\hline PECF & 145 & 5 & - & - & 30 & 118 & 1 & - \\
\hline
\end{tabular}

\section{Incubation studies}

In the incubation studies $0.5 \mathrm{~g}$ of prepared acrylic hydrogels were placed in $50 \mathrm{ml}$ of an appropriate medium: water, SBF and Ringer's solution for a period of 32 days. During this time $\mathrm{pH}$ values were measured periodically.

\section{Attenuated total reflectance FT-IR spectroscopy analysis}

All Attenuated Total Reflectance Fourier-Transform Infra-Red (ATR-FT-IR) spectra were recorded using Spectrum 65 (Perkin Elmer) spectrometer. The ATR probe was equipped with a diamond crystal. All spectra consisted of 32 scans at $4.0 \mathrm{~cm}^{-1}$ resolution and were recorded at $25^{\circ} \mathrm{C}$.

\section{Scanning Electron Microscopy}

In order to investigate the structure of the obtained hydrogels modified with bee pollen, as well as bee pollen alone, images using scanning electron microscopy were taken and analyzed (JEOL JSM 7500F with a Back Scattered Electrons detector).

\section{RESULTS AND DISCUSSION}

\section{SEM analysis}

In order to assess whether and how the hydrogel and bee pollen interact, SEM images of both bee pollen and the hydrogel modified with bee pollen were taken. Fig. 1 shows bee pollen in its raw form, as purchased. As it can be seen, bee pollen grains occur mostly in elliptic forms with two or three grooves. Moreover, these particles have a very uniform size distribution. This is due to the fact that the pollen grains were all collected from the same plant species.

Enlargement of a single particulate of bee pollen clearly shows a porous surface of the grain, with a very uniform distribution of the pores. Since the surface is not smooth but exhibits certain porosity, it is highly likely that adhesion to such a surface ought to be feasible and allow good bonding between the acrylic hydrogel matrix and the natural substance (see Fig. 1).

Images shown in Fig. 2, were collected using SEM imaging and show hydrogel modified with bee pollen. The image to the left shows fragments of the gel in reasonably small magnification, while the image in the centre shows clearly how the bee pollen particles are bound to the hydrogel. As expected, it appears that the gel at least partially coats the bee pollen particles. 

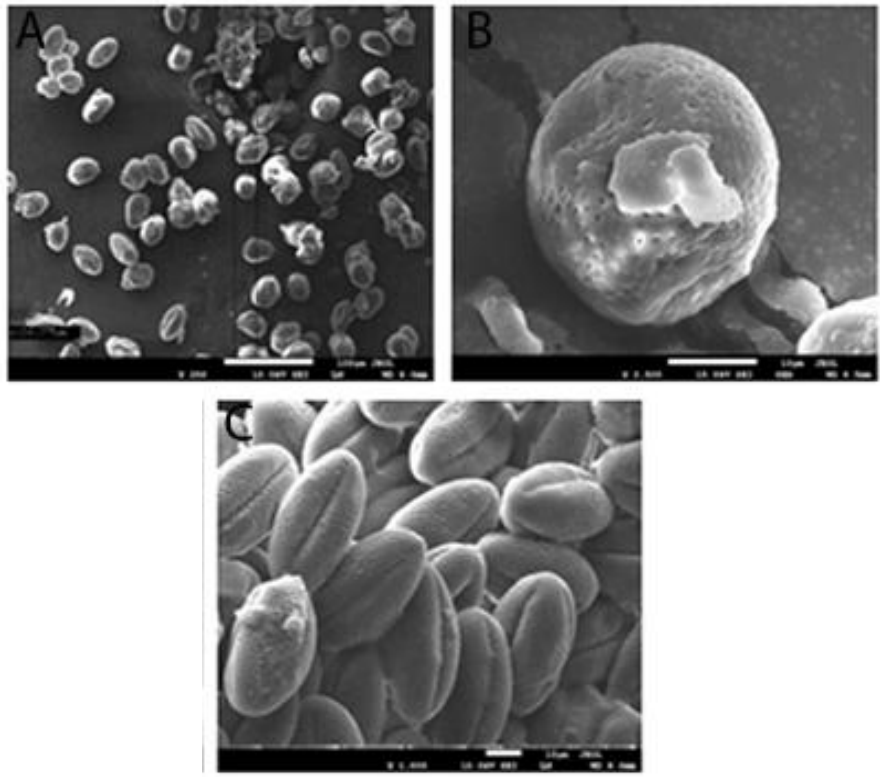

Fig. 1: SEM images of bee pollen where A denotes raw bee pollen magnified250x, B shows single particulate of raw bee pollen magnified 2500x and C-shows enlargement of image A magified1000x.
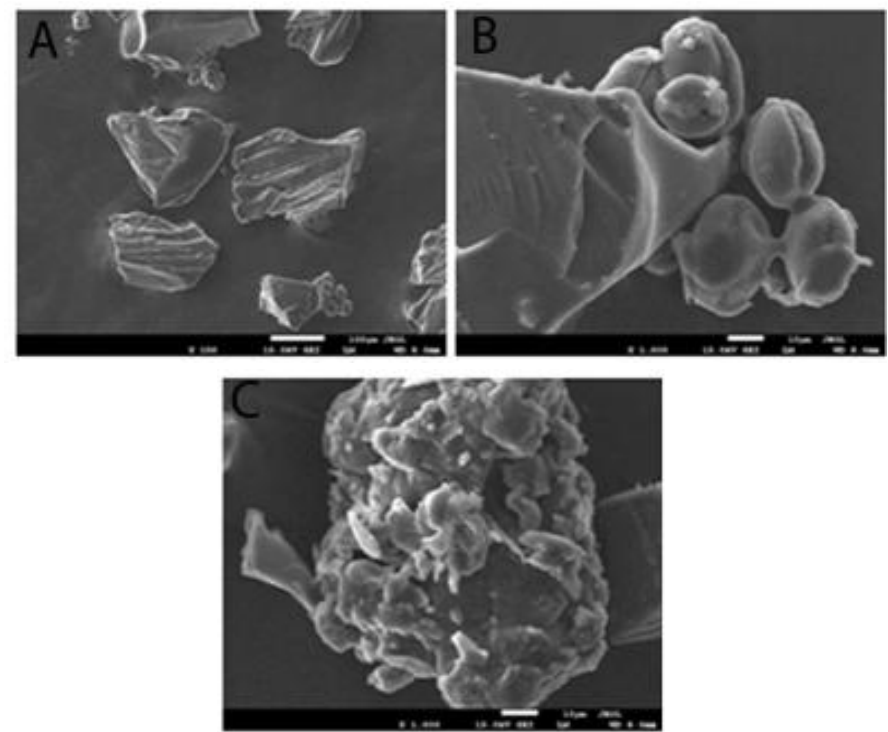

Fig. 2: SEM micrographs of acrylic hydrogels modified with bee pollen where A denotes acrylic hydrogel modified with bee product magnified 150x, B- and C- single particulate of modified hydrogel magified 1000x.

\section{Swelling behavior}

Hydrogels are very well known for their excellent moisture sorption abilities which allow them to be used in a variety of biomedical and personal hygiene applications. Addition of any substance, such as bee pollen, may affect these properties therefore sorption capacity of modified hydrogel was tested using a variety of solutions, as it is shown in Fig.

The results show that the polymer matrix and modified pollen hydrogels exhibit the lowest sorption capacity in $\mathrm{CaCl}_{2}$ solution. This is a consequence of presence of $\mathrm{Ca}^{2+}$, a multivalent cation which contributes to further cross-linking of the gel, leaving less void spaces in the macromolecule three-dimensional structure available for the solution to penetrate. The highest sorption values are achieved upon sorption of water, as expected. The water molecules can easily penetrate to the interior of the hydrogel and since they are not charged and are inert, they do not meet any hindrances.

It is impossible to define an overall relationship between the amount of added bee pollen and general sorption capacity. In case of swelling in SBF, addition of bee pollen caused a dramatic decrease, while other sorption capacities changed only insignificantly and could be both positive (PECF, Ringer's solution, water) and negative $(0.9 \% \mathrm{wt}$. $\mathrm{NaCl})$. It is possible that the worsened sorption capacities are caused by the fact that there is less volume available to penetrate in the hydrogel matrix, as it is partially occupied by the bee pollen particles. Nevertheless, since sorption properties are not severely affected, there is a good possibility that hydrogel modified with bee pollen could be used in the same applications as the non-modified material.

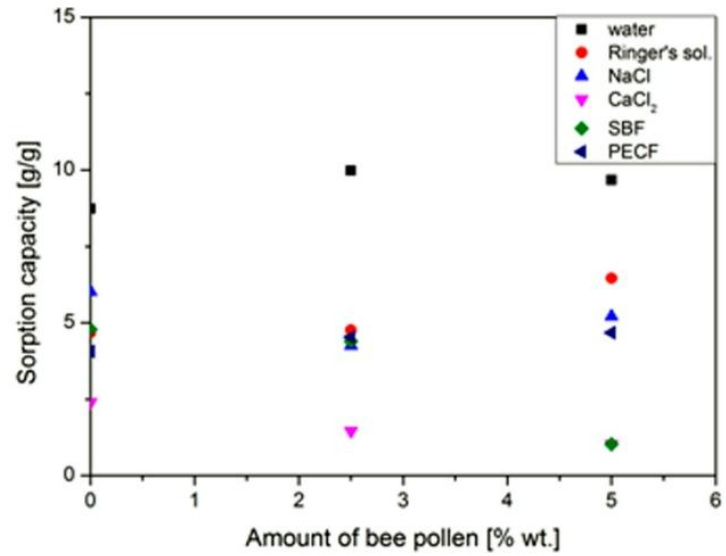

Fig. 3: Sorption capacity of acrylic hydrogel modified with bee pollen.

\section{Incubation studies}

In order to assess stability of the obtained hydrogels modified with bee pollen, $\mathrm{pH}$ measurements were carried out in the course of 32 days of incubation in the fluids mentioned in the Experimental section above. The results of these measurements performed on hydrogel modified with $2.5 \%$ wt. and $5 \%$ wt. of bee pollen are shown in Fig. 4 a and b, respectively.

The conducted $\mathrm{pH}$ measurements during the incubation period show significant changes in $\mathrm{pH}$ values only for the first four days, after which, regardless of the type of solution used, a plateau is reached. In case of Ringer's fluid and SBF, this initial change which took place over the first four days is far more significant than for samples immersed in water. This is certainly associated with presence of metal cations in both SBF and Ringer's solution and their absence in de-ionized water. As the results show, amount of introduced bee pollen has no significant effect on the $\mathrm{pH}$ changes during the incubation. The results for both polymer matrices containing $2.5 \% \mathrm{wt}$. and $5 \% \mathrm{wt}$. of bee pollen, are very similar. Lack of changes over an extended period of time appears to confirm that the hydrogel matrix would not degrade in conditions typical for biomedical applications. 

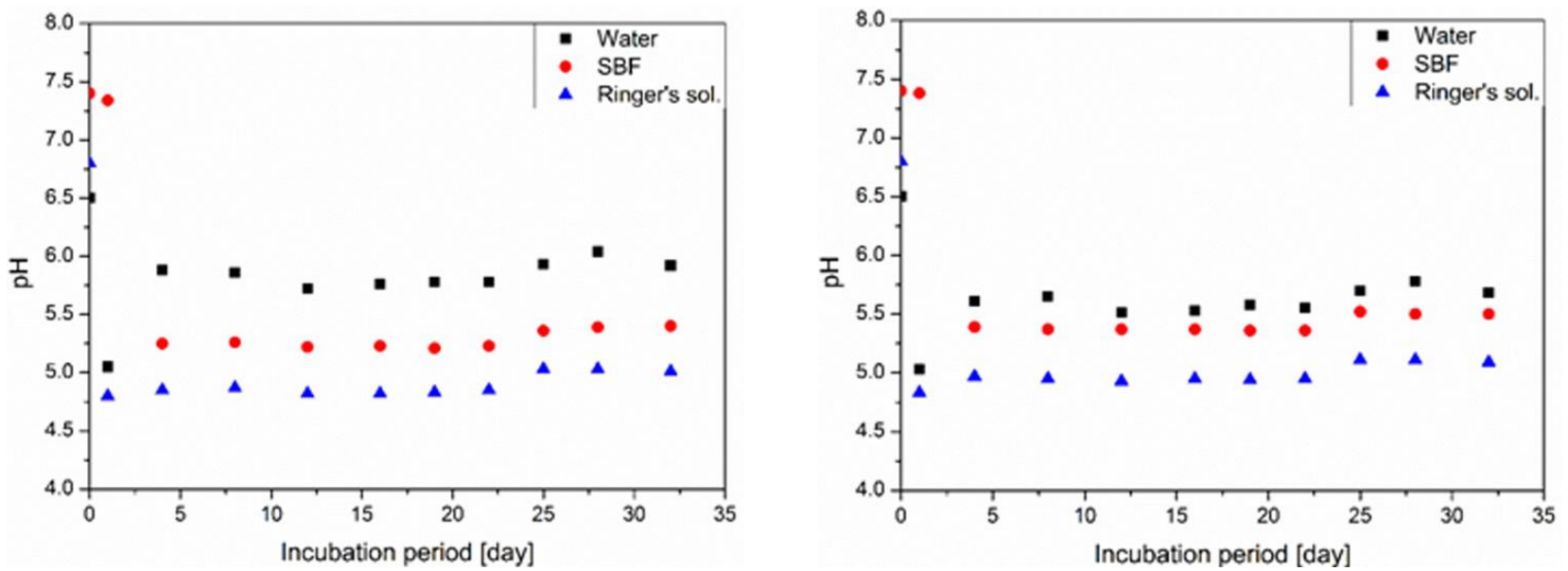

Fig. 4: Results of incubation studies, expressed as $\mathrm{pH}$ variation, of hydrogel matrices modified with $2.5 \%$ wt. bee pollen (a) and $5 \%$ wt. bee pollen (b).

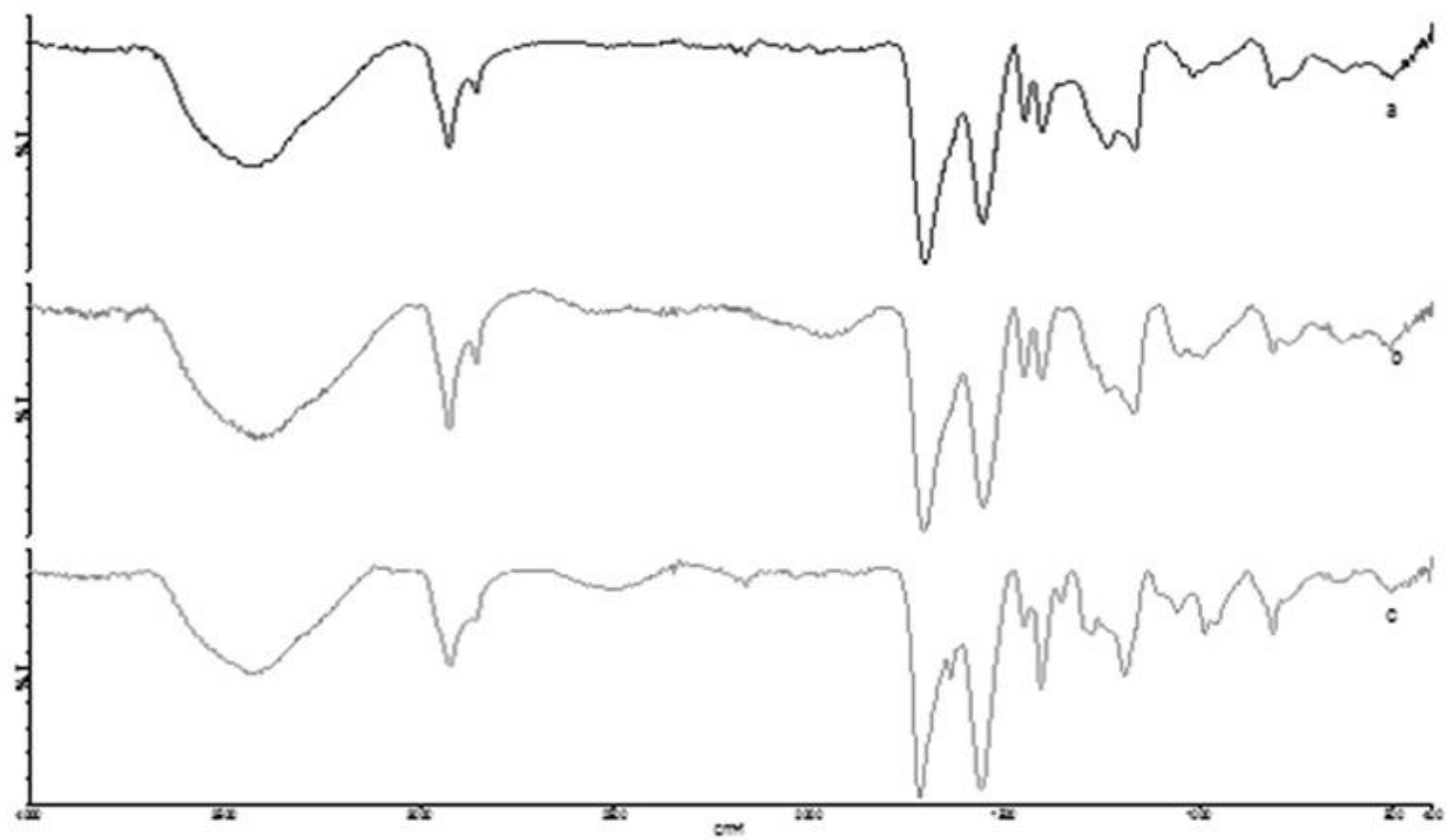

Fig. 5: The ATR-FT-IR spectra of acrylic hydrogels containing a) $0 \%$ wt., b) $2.5 \%$ wt. and c) $5 \%$ wt. bee pollen.

\section{Attenuated total reflectance FT-IR spectroscopy analysis}

The structure of the hydrogel matrix and presence of functional binding groups such as ester and ether groups, as well as carbonyl, hydroxyl and carboxyl groups have been confirmed by means of Attenuated Total Reflectance Fourier-Transform Infra-Red (ATR-FT-IR) spectroscopy. The resulting spectra are shown in Fig. 5 above. In all spectra a wide signal is observed in the range of $3700-3100 \mathrm{~cm}^{-1}$, and it is resulting from stretching vibrations of hydroxyl groups and hydrogen interactions (formation of hydrogen bonds with hydroxyl groups, most likely). Presence of $-\mathrm{CH}_{2}$ - groups is confirmed by the signal found at $2930-2900 \mathrm{~cm}^{-1}$, resulting from valence vibrations of these groups. The band from the range of $1553-1556 \mathrm{~cm}^{-1}$ corresponds to the vibrations of the carbonyl group of poly (acrylic acid).
A signal caused by presence of ester groups of poly(ethylene glycol) diacrylate is observed at around $1704-1717 \mathrm{~cm}^{-1}$. Signals corresponding to symmetrical scissor mode vibrations of the $-\mathrm{CH}_{2}-$ groups are observed at a wavelength of ca. $1450 \mathrm{~cm}^{-1}$. The structure of PEGDA is confirmed by peaks corresponding to C-O-C stretching, which are visible at 1165 and $1014 \mathrm{~cm}^{-1}$. Furthermore, in case of acrylic hydrogels modified by $5 \%$ wt. of bee pollen several subtle changes in the overall shape of the spectrum in the range of $1405-800 \mathrm{~cm}^{-1}$ are observed.

For example, C-O-C stretching vibrations from PEGDA were observed at different wavelengths, i.e. 1190 and $1045 \mathrm{~cm}^{-1}$. In addition, an extra peak at $985 \mathrm{~cm}^{-1}$ is observed and can be assigned to the $\mathrm{C}-\mathrm{OH}$ out-of-plane bending and $-\mathrm{CH}_{2}$ - twisting modes (Silverstein et al., 2014). 


\section{CONCLUSIONS}

The focus of this research was to obtain hydrogel acrylic matrices modified with bee pollen and to pre-evaluate their potential for biomedical applications. Synthesis proposed by the authors is straightforward and allows to obtain hydrogel matrices in which bee pollen particles are well distributed and bound to the acrylic material. The resulting materials exhibit good sorption capacity against different liquids, typically used in biomedical testing, such as Ringer's solution, SBF, PECF. Series of incubation experiments proved that these materials are stable in body-simulated conditions, as well as in conditions mimicking wound environment, over an extended period of time. Bearing in mind that acrylic hydrogels are already in use as wound dressing materials, further tests should focus on the benefits of addition of bee pollen into hydrogel matrices. It is expected that such modified dressings would have anti-inflammatory and antiseptic properties, and would also enhance the healing time of the wound.

\section{REFERENCES}

Almeida-Muradian, LB, Lucila C Pamplona , Sílvia Coim bra, and Ortrud Monika Barth. Chemical composition and botanical evaluation of dried bee pollen pellets. Journal of food composition and analysis, 2005; 18 (1):105-111.

Burdock, GA. Review of the biological properties and toxicity of bee propolis (propolis). Food and Chemical toxicology, 1998; 36 (4):347-363.

Catchpole, Owen J, JB Grey, KA Mitchell, and JS Lan. "Supercritical antisolvent fractionation of propolis tincture. The Journal of supercritical fluids, 2004; 29 (1):97-106.

Human, Hannelie, and Sue W Nicolson. Nutritional content of fresh, bee-collected and stored pollen of Aloe greatheadii var. davyana (Asphodelaceae). Phytochemistry, 2006; 67 (14):1486-1492.

Kashkooli, Omid Beyraghdar, Eisa Ebrahimi Dorcheh, Nasrollah Mahboobi-Soofiani, and Abdolhossein Samie. Long-term effects of propolis on serum biochemical parameters of rainbow trout (Oncorhynchus mykiss). Ecotoxicology and environmental safety, 2011; 74 (3):315-318.

Kokubo, Tadashi, and Hiroaki Takadama. How useful is SBF in predicting in vivo bone bioactivity? Biomaterials, 2006; 27 (15):29072915.

Kroyer, G, and N Hegedus. Evaluation of bioactive properties of pollen extracts as functional dietary food supplement. Innovative Food Science \& Emerging Technologies, 2001; 2 (3):171-174.

Leja, M, A Mareczek, G Wyżgolik, J Klepacz-Baniak, and K Czekońska. Antioxidative properties of bee pollen in selected plant species. Food Chemistry, 2007; 100 (1):237-240.

Peppas, Nicholas A, and J Zach Hilt. Hydrogels in biology and medicine: from molecular principles to bionanotechnology. ADVANCED MATERIALS-DEERFIELD BEACH THEN WEINHEIM-, 2006; 18(11), 1345

Queen, Douglas, Heather Orsted, Hiromi Sanada, and Geoff Sussman. A dressing history. International wound journal, 2004; 1 (1):5977.

Serra, Laura, Josep Doménech, and Nicholas A Peppas. "Engineering design and molecular dynamics of mucoadhesive drug delivery systems as targeting agents. European journal of pharmaceutics and biopharmaceutics, 2009; 71 (3):519-528.

Sforcin, JM. Propolis and the immune system: a review. Journal of ethnopharmacology, 2007; 113 (1):1-14.

Sforcin, José Maurício and Vassya Bankova. Propolis: is there a potential for the development of new drugs? Journal of ethnopharmacology, 2011; 133 (2):253-260.

Silverstein, Robert M, Francis X Webster, David Kiemle, and David L Bryce. 2014, Spectrometric identification of organic compounds: John Wiley \& Sons.

Vallet-Regí, María, and Daniel Arcos. 2008. Biomimetic nanoceramics in clinical use: from materials to applications: Royal Society of Chemistry.

\section{How to cite this article:}

Tyliszczak B, Walczyk D, Wilczynski S. Acrylic hydrogels modified with bee pollen for biomedical applications. J App Pharm Sci, 2015; 5 (11): 010-014. 